\title{
La Bible d'Étienne Harding et les origines de Cîteaux : perspectives de recherche
}

\section{Alessia Trivellone}

\section{(2) OpenEdition}

1 Journals

\section{Édition électronique}

URL : https://journals.openedition.org/cem/11101

DOI : 10.4000/cem. 11101

ISSN : 1954-3093

Éditeur

Centre d'études médiévales Saint-Germain d'Auxerre

\section{Édition imprimée}

Date de publication : 15 août 2009

Pagination : 303-319

ISSN : 1623-5770

\section{Référence électronique}

Alessia Trivellone, "La Bible d'Étienne Harding et les origines de Cîteaux : perspectives de recherche», Bulletin du centre d'études médiévales d'Auxerre / BUCEMA [En ligne], 13 | 2009, mis en ligne le 10 février 2010, consulté le 22 septembre 2022. URL : http://journals.openedition.org/cem/11101 ; DOI : https:// doi.org/10.4000/cem. 11101

Ce document a été généré automatiquement le 22 septembre 2022.

\section{(c) (1) (ㅇ)}

Creative Commons - Attribution - Pas d'Utilisation Commerciale - Partage dans les Mêmes Conditions 4.0 International - CC BY-NC-SA 4.0

https://creativecommons.org/licenses/by-nc-sa/4.0/ 


\title{
La Bible d'Étienne Harding et les origines de Cîteaux : perspectives de recherche
}

\author{
Alessia Trivellone
}

1 La Bible dite "d'Étienne Harding " (Dijon, BM, ms 12 à 15) est l'un des manuscrits médiévaux les plus connus des médiévistes. Aujourd'hui en quatre volumes, la Bible était formée au départ par deux tomes : le premier correspondait aux deux premiers volumes actuels (cotes 12 et 13) et le deuxième regroupait les troisième et quatrième volumes d'aujourd'hui (cotes 14 et 15). Le premier tome originel se termine par un Monitum (ms 13, fol. 150), à savoir un «avertissement » dans lequel Étienne Harding, d'origine anglaise, abbé de Cîteaux entre 1108 et 1133 (†1134), explique quelques circonstances liées à la réalisation du manuscrit. Le Monitum étant daté de 1109, on peut conclure qu'à cette date le premier volume de la Bible était terminé. Le deuxième volume, en revanche, a été complété, au plus tard, deux ans après. Le scribe qui l'a copié est en effet le même que celui du premier volume du manuscrit des Moralia in Job (Dijon, ms 168 à 170 et ms 173), qu'un colophon permet de dater de $1111^{1}$. Par sa datation entre 1109 et 1111, la Bible est ainsi l'un des premiers témoignages, sinon le tout premier produit, du scriptorium de l'abbaye de Cîteaux ${ }^{2}$.

2 En 1098, le Novum Monasterium, plus tard appelé Cistercium (Cîteaux) ${ }^{3}$, est fondé par un groupe de moines provenant de Molesmes souhaitant appliquer dans la nouvelle abbaye un retour à la règle bénédictine authentique. Or, certains aspects de la réalisation de la Bible d'Étienne Harding répondent également à un désir de refondation et de retour aux origines. Tout d'abord, remarquons que la Bible, qui n'est pas un livre ordinaire, mais le livre fondamental du christianisme, est, à l'époque, l'un des premiers textes que les communautés monastiques de récente fondation se doivent de posséder. Cette nécessité répondait certes à des raisons pratiques, car la Bible avait une large place dans la liturgie monastique et était lue, dans les monastères, à différents moments de la journée: dans la Bible d'Étienne Harding même, une note marginale du XII ${ }^{e}$ siècle, au fol. 166v du ms 14 (Hic incipe legere ad mensam), indique que 
le passage voisin ( $\mathrm{Tb} 3,16$ et suiv.) était lu pendant les repas ${ }^{4}$. L'effort souvent fait pour la réalisation et la décoration des manuscrits contenant l'Écriture témoigne toutefois également de l'importance symbolique de ces manuscrits ${ }^{5}$. La réalisation de la Bible d'Étienne Harding, manuscrit enrichi de nombreuses miniatures, est d'autant plus étonnante qu'elle se fait dans les premiers temps de la vie de l'abbaye, dans une période peu fleurissante du point de vue économique ${ }^{6}$. Cette Bible naît donc lors des premières années de vie de Cîteaux et sa réalisation inaugure, très probablement, l'activité du scriptorium. Des éléments soulignent davantage le désir de refondation lié à la réalisation de ce manuscrit. Étienne Harding nous explique, en effet, dans son Monitum, qu'après avoir entrepris la copie de la Bible, il s'est consacré à une révision de son texte: ayant constaté de nombreuses discordances dans les copies disponibles, il se proposait de revenir à la version textuelle la plus proche de la Vulgate de Jérôme. Il déclare ensuite avoir accompli cette tâche en recourant à une comparaison avec le texte hébraïque, faite grâce à des juifs «expérimentés dans leur langue et dans leurs écritures " ${ }^{7}$. Ce travail de révision du texte biblique s'accorde à ce désir général de « retour à la pureté des origines » qui anime l'expérience des premiers cisterciens et, qui, depuis quelques décennies, inspirait plus largement la hiérarchie ecclésiastique engagée dans la réforme ecclésiastique ${ }^{8}$.

3 La Bible d'Étienne Harding reflète donc, à différents niveaux, le climat de refondation et de réforme qui accompagne la création de Cîteaux, en incarnant les valeurs et les aspirations de la première communauté monastique et devenant ainsi un témoignage précieux sur les origines de l'abbaye, dont l'histoire primitive demeure encore méconnue ${ }^{9}$. À partir de ces prémisses, nos recherches post-doctorales à l'université de Bourgogne, au sein de l'UMR ARTeHIS 5594, financées par la Région Bourgogne pour l'année 2008-2009, envisagent d'étudier les miniatures de ce manuscrit : bien étudiées d'un point de vue stylistique ${ }^{10}$, elles n'ont pas encore fait l'objet d'une étude iconographique d'ensemble ${ }^{11}$. Nous nous proposons ainsi de combler cette lacune, en considérant ces miniatures comme des sources à part entière. L'étude iconographique se propose d'éclairer quelques aspects de l'esprit cistercien des origines et de l'histoire primitive de Cîteaux.

4 La recherche se fonde sur le principe selon lequel le choix des sujets représentés, ainsi que l'originalité des formules iconographiques utilisées, ont une signification intrinsèque ${ }^{12}$. Tout d'abord, dans le cadre de l'illustration biblique, la décision de représenter certains sujets plutôt que d'autres, au sein du vaste choix offert par le récit biblique, nous paraît significative en elle-même. Ensuite, les nombreux manuscrits bibliques enluminés des $\mathrm{XI}^{\mathrm{e}}$ et $\mathrm{XII}{ }^{\mathrm{e}}$ siècles ne présentent jamais exactement ni les mêmes scènes, ni les mêmes formules iconographiques ${ }^{13}$. Les différences entre les diverses formules employées pourraient être dues, dans certains cas, à la volonté de mieux adapter l'image à l'idée que l'on veut véhiculer. En comparant quelques-unes de nos miniatures avec celles d'autres Bibles médiévales enluminées, l'originalité des illustrations de la Bible d'Étienne Harding ressort de manière évidente. Les études iconographiques menées sur quelques images de ce manuscrit ont déjà démontré la prégnance des formules iconographiques choisies ${ }^{14}$.

5 La réflexion sur ces illustrations est susceptible de fournir de riches opportunités de découvrir la vision de la société dans son milieu de réalisation. La Bible est en effet, au Moyen Âge, la clé pour expliquer ou justifier de nombreux aspects de la vie sociale. Tout comme l'étude de l'exégèse biblique médiévale se révèle un instrument utile à la 
recherche historique politique ${ }^{15}$, les miniatures de la Bible, considérées comme une forme d'interprétation du texte biblique, sont ainsi susceptibles de nous renseigner sur la vision de la société dans leur contexte de réalisation. Des études sur d'autres Bibles enluminées ont été d'ailleurs faites suivant cette perspective. Parmi celles-ci, signalons celle récente de Diane J. Reilly, qui se propose, à partir de l'étude de la Bible de SaintVaast d'Arras, de reconstruire la perception de la contemporanéité chez les familles royales et les créateurs religieux des images, ainsi que les relations entre la Bible et le contexte de la Réforme ${ }^{16}$. Dans notre recherche sur la Bible d'Étienne Harding, nous éviterons en revanche l'approche adoptée dans certaines études sur ces miniatures, qui, tout en se proposant d'examiner les reflets de l'histoire du XII siècle dans les images, s'attachent aux aspects les plus superficiels des représentations et finissent par se focaliser sur la mode vestimentaire de l'époque, les rites de la vie courtoise, etc. ${ }^{17}$. Il s'agira, au contraire, de réfléchir sur le sens profond des images, en prenant en compte, d'un côté, leur emplacement par rapport au texte et aux autres enluminures, et, d'un autre côté, les éléments originaux des formules iconographiques employées. Ces particularités seront à comprendre également grâce à la comparaison avec les sources textuelles, qui nous permettra d'insérer ces miniatures au sein de la vie de l'abbaye et de ses relations avec le monde extérieur ${ }^{18}$.

Après ces considérations d'ordre méthodologique, des réflexions sur la structure de l'apparat décoratif du manuscrit, ainsi que l'analyse d'une image de la Bible tirée de l'incipit de l'Évangile de Jean, seront utiles à illustrer nos propos.

\section{Un « discours visuel » cohérent ?}

7 La réalisation matérielle du manuscrit, telle que Yolanta Załuska l'a reconstruite, couvre une période d'au moins deux ans et voit la succession de trois copistes et d'au moins deux enlumineurs, dont l'un aurait réalisé les initiales du premier volume originel (ms 12 et 13 ) et l'autre les miniatures du deuxième (ms 14 et 15). Cette reconstruction prouve que l'ensemble des illustrations de la Bible, tel qu'on peut le voir aujourd'hui, ne répond pas à un projet unitaire, conçu dès le début, mais qu'il est plutôt le résultat des deux phases de réalisation et de quelques corrections en cours d'œuvre. Il nous semble important de remarquer, toutefois, que ces modifications pourraient répondre plutôt à l'intention de rendre à l'ensemble des miniatures de la Bible une stricte cohérence. Le feuillet 13 du manuscrit 14 constitue un indice de cette intention. Il s'agit d'un singulion, entièrement occupé par des enluminures concernant David, avec, au recto, une séquence narrative sur cinq registres superposés (fig. 1) et, au verso, une représentation de David jouant de sa harpe au milieu de murailles (fig. 2). Le feuillet fut ajouté en tête du psautier après l'écriture de celui-ci, très probablement en même temps qu'on ajoutait, au début du manuscrit, le livre de Job, copié sur un quaternion amputé du premier feuillet (fol. 2-8) et un binion (fol. 9-12), par un scribe différent de celui du psautier ${ }^{19}$. La présence du feuillet avec les deux enluminures en pleine page du folio 13 n'est évidemment pas motivée par la nécessité de corriger le texte. Elle ne peut donc que répondre à la volonté de mettre ces images consacrées à David à l'emplacement voulu. L'acte d'insérer ce feuillet souligne donc l'importance de ces images au sein du manuscrit. Elles doivent nécessairement y apparaître à cet emplacement précis. Cette insertion implique donc l'existence d'un discours visuel qui se fait à travers les enluminures du manuscrit. 
Fig. 1 - Dijon, Bibliothèque municipale, Ms 14, fol. 13 (d'après Zaluska, Manuscrits enluminés, 1991).

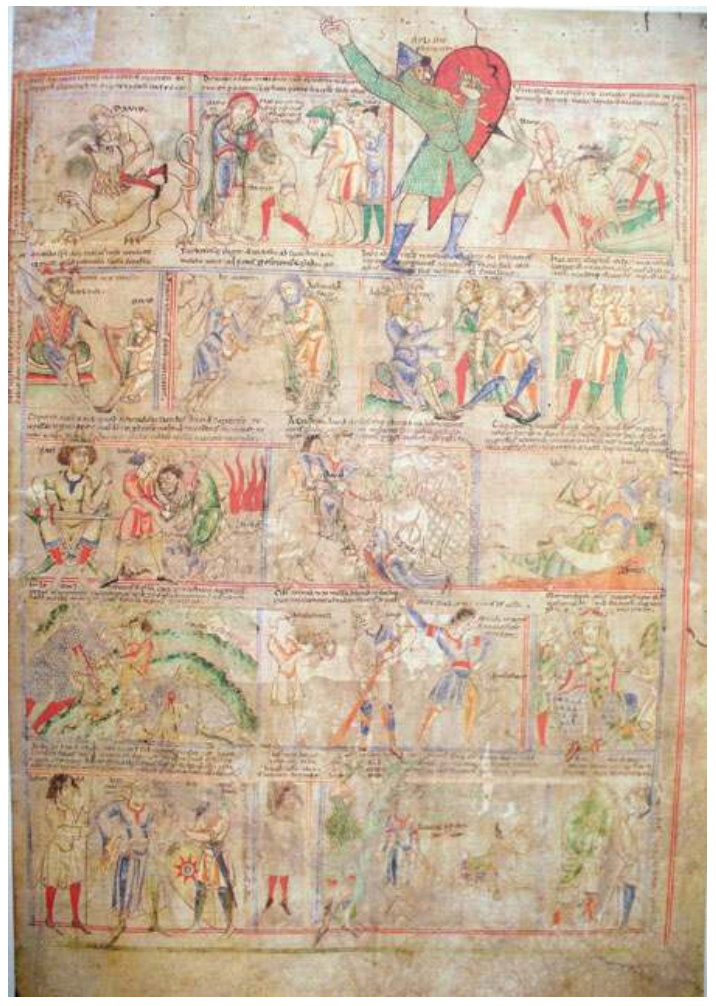

Fig. 2 - Dijon, Bibliothèque municipale, Ms. 14, fol. 13v (d'après Voix enluminées de Cîteaux, 1998).

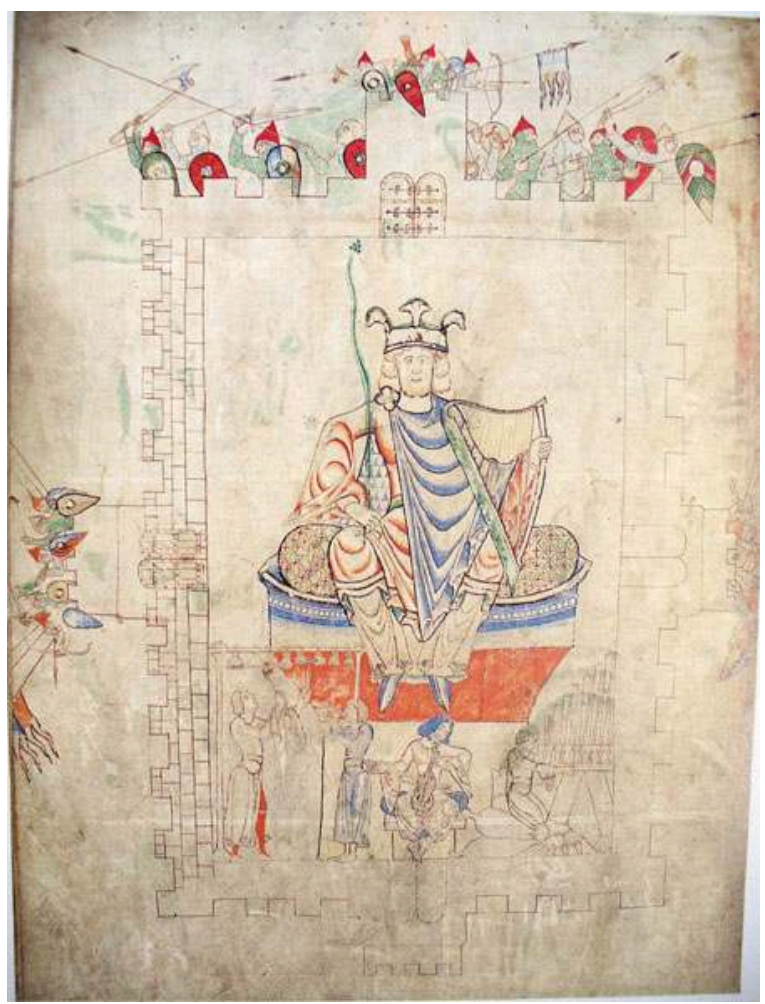


8 L'insertion de ce feuillet apparaît d'autant plus intéressante qu'il contient dans la succession des feuillets les premières scènes historiées de la Bible. Il est toutefois intéressant de porter l'attention sur la toute première scène du folio 13r, qui est donc la première scène historiée de la Bible. Elle représente David qui tue le lion (1 Sm 17, 34-36, fig. 3). Comme on l'a fait remarquer, la présence de cette image au début de la séquence des scènes ne respecte pas l'ordre du récit biblique. Le combat entre David et le lion n'est en effet qu'un épisode rétrospectif que David raconte lors de sa seconde arrivée à la cour de Saül. Certes, l'emplacement au début des scènes se justifierait si l'on admettait que la séquence veut respecter l'ordre chronologique de la biographie de David et non pas du récit biblique ${ }^{20}$. Il n'en demeure pas moins, toutefois, que cette scène est normalement ignorée par les cycles des illustrations bibliques ${ }^{21}$. Sa présence pourrait mieux s'expliquer, nous semble-t-il, en relation avec les miniatures du premier volume de la Bible. Celles-ci, réalisées par un artiste différent de celui du deuxième volume, comprennent uniquement des initiales formées par des rinceaux végétaux, où des animaux (cervidés, canidés, hybrides) et, dans un cas ( $m s$ 13, fol. 132v), une tête humaine, apparaissent, parfois presque hybridés dans les végétaux - cf. par exemple le $\mathrm{ms} 12$, fol. 42 - ou comme prisonniers dans les entrelacs. Des rinceaux végétaux, comme ceux représentés dans ces initiales, sont parmi les motifs les plus communs de l'art médiéval et se retrouvent sur tous les supports, de l'enluminure à la sculpture monumentale ou à l'orfèvrerie. Jadis considérés comme des drôleries, la critique récente commence à accorder à ce type de décor une plus grande attention et, dans différents cas, en a décrypté la signification. Il est intéressant pour notre propos de constater que la végétation et les animaux sauvages sont souvent considérés, notamment dans le cadre du motif du « rinceau peuplé », comme une allusion à la forêt et sont ainsi souvent interprétés comme une allusion à la nature sauvage. Les figures humaines souvent présentes dans les entrelacs représenteraient l'Homme qui lutte contre la nature de ses passions pour atteindre la rédemption ${ }^{22}$. Dans le cas précis de la Bible d'Étienne Harding, la lutte de l'Homme contre la nature n'est pas directement évoquée par des figures humaines imbriquées dans les entrelacs : une image comme celle du cerf pris dans la végétation et attaqué par un chien, dans le manuscrit 13, au folio 2 (fig. 4), peut toutefois facilement avoir la même signification, compte tenu de la traditionnelle association de la figure du cerf à l'âme humaine. 
Fig. 3 - Dijon, Bibliothèque municipale, Ms. 14, fol. 13v, détail (d'après Voix enluminées de Cîteaux, 1998).

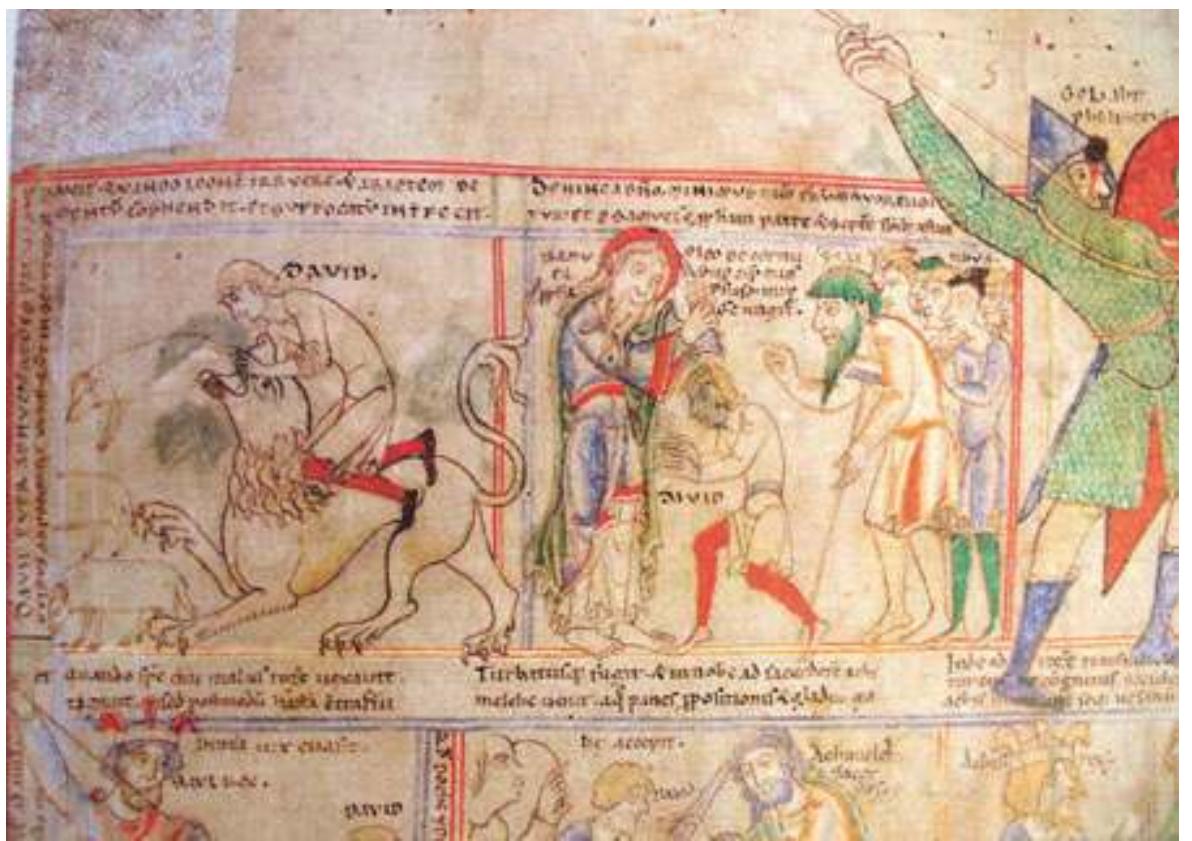

Fig. 4 - Dijon, Bibliothèque municipale, Ms. 13, fol. 2 (photo auteur).

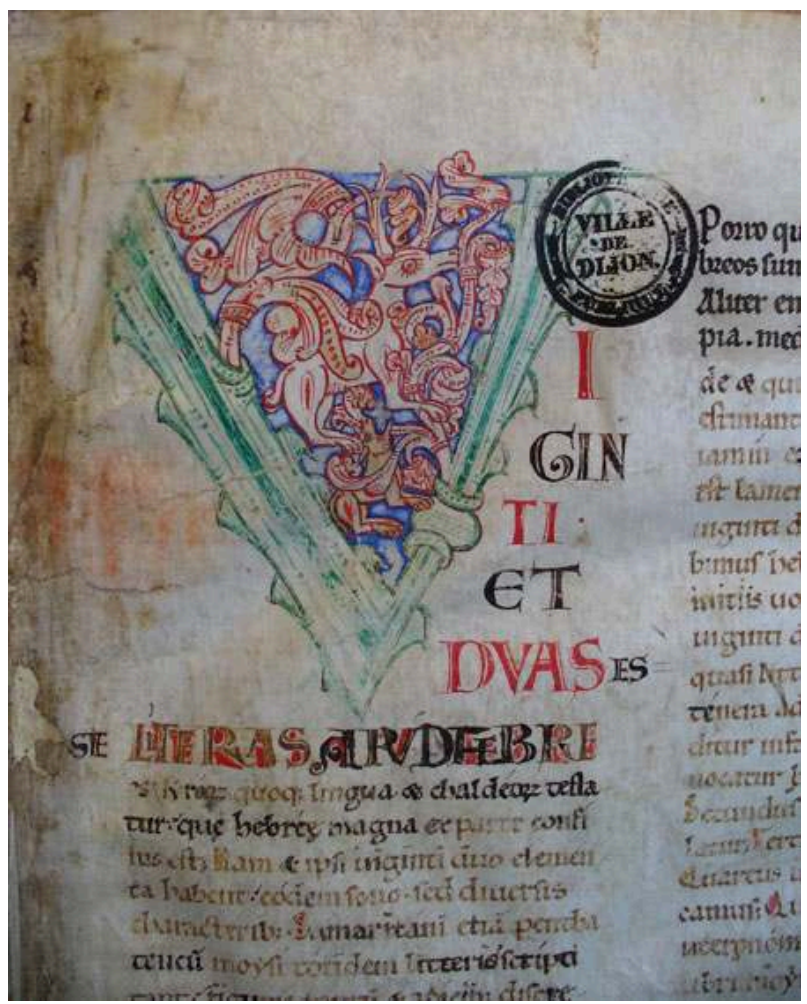

9 Dans cette perspective, le choix de la lutte de David contre le lion pour ouvrir le discours iconographique du deuxième tome, pourrait trouver une autre explication. Il introduit en effet une forme de continuité ou de contrepoint aux illustrations du premier volume. David abattant le lion est en effet le symbole même, dans plusieurs situations, de l'Homme, qui, luttant contre l'animalité, arrive à la maîtriser et à la 
dominer ${ }^{23}$. De ce point de vue, il est possible que la miniature vise à renouer, du point de vue du sens, la continuité avec les miniatures du volume précédent et à introduire le nouveau récit, qui se déroule à travers les scènes, en large partie historiées, de l'ancien deuxième volume de la Bible ${ }^{24}$.

D'autres miniatures semblent également avoir une signification en rapport avec leur emplacement à l'intérieur du manuscrit et en relation avec les autres enluminures : ainsi, par exemple, la toute dernière image du deuxième volume originaire de la Bible représente le triomphe de l'archange Michel sur le dragon (aujourd'hui ms 15, fol. 125 ; fig. 5). Cette lutte semble proposer sous une forme plus avancée l'idée de lutte évoquée par David et le lion : elle semble de plus marquer le passage d'une lutte terrestre à une lutte eschatologique (Ap 12, 7). L'image de triomphe qui en suit apparait particulièrement adéquate pour terminer la série des illustrations bibliques.

Ces détails sont des indices de l'existence d'un discours visuel cohérent mis en place en cours d'œuvre ou, au plus tard, immédiatement après l'achèvement de la copie, et qui se déroule à travers les deux volumes. Un des buts de notre recherche sera de préciser le contenu de ce discours.

Fig. 5 - Dijon, Bibliothèque municipale, Ms. 15, fol. 125 (d'après Oursel, Miniatures cisterciennes, 1960).

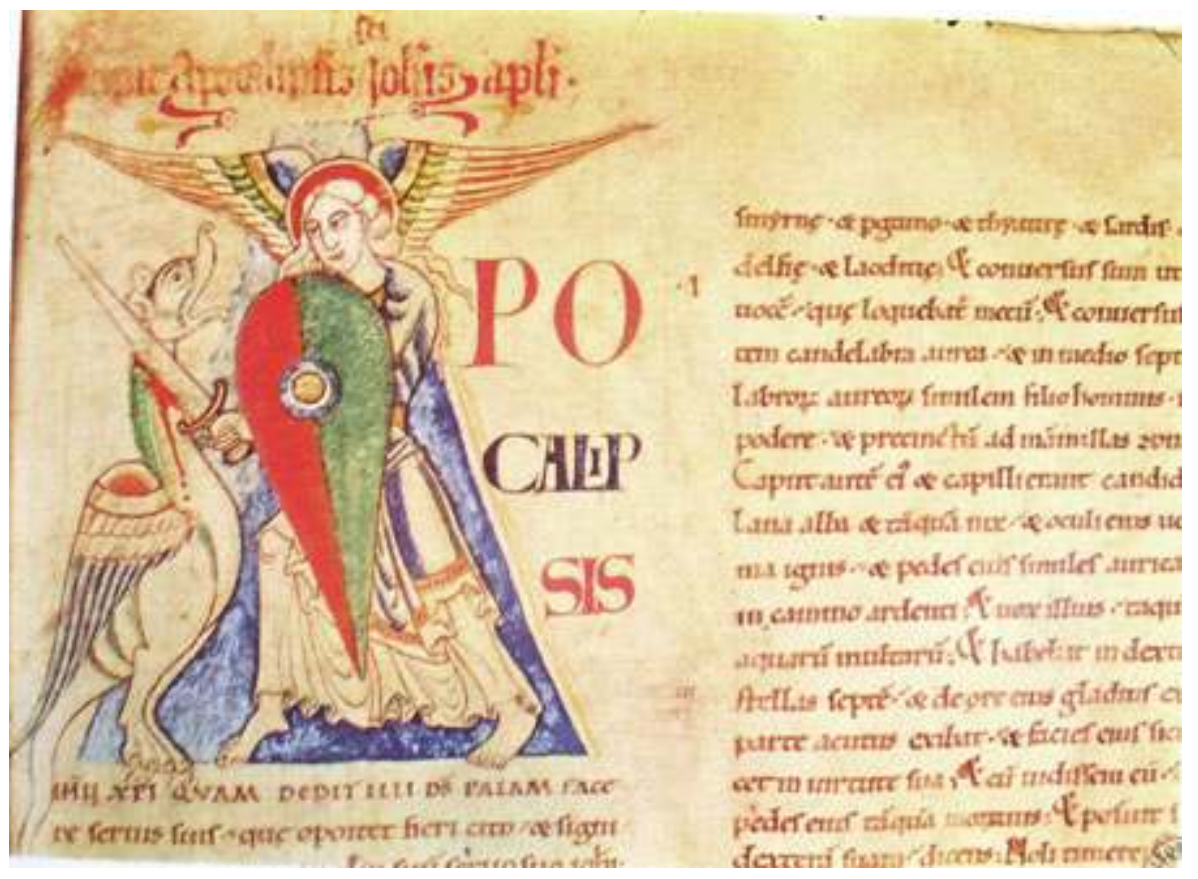

\section{La prégnance des créations iconographiques : l'exemple de l'enluminure de Jean et Arius (ms 15, fol. 56v)}

L'originalité de quelques formules iconographiques a été mise en avant par différentes études ponctuelles ${ }^{25}$. Nous nous limiterons, dans le cadre de cette brève présentation, à analyser la miniature du manuscrit, qui, jusqu'à présent, a attiré le plus l'attention des 
spécialistes, à savoir l'image accompagnant l'incipit de l'Évangile selon Jean (ms 15, fol. 56v ; fig. 6).

Fig. 6 - Dijon, Bibliothèque municipale, Ms. 15, fol. 56v (d'après Oursel, Miniatures cisterciennes, 1960).

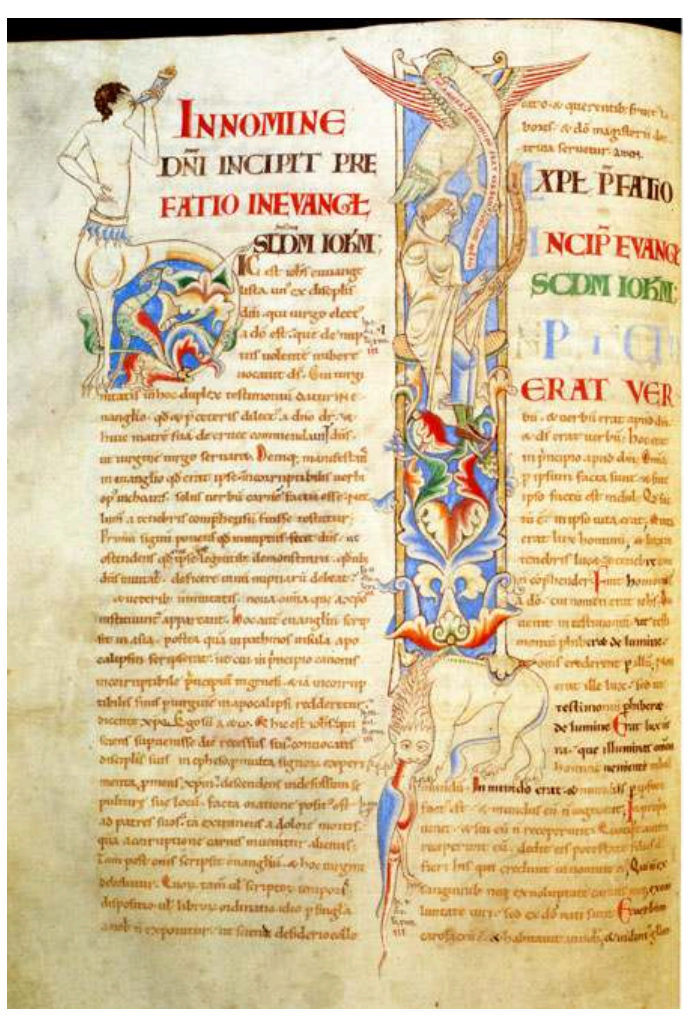

L'enluminure montre l'aigle de saint Jean saisissant dans ses griffes la tête d'un moine assis et vêtu d'une tunique, indiquant de son doigt un phylactère avec la proposition hérétique d'Arius condamnée au concile de Nicée. Les spécialistes se sont divisés sur l'identité du personnage saisi par les griffes de l'aigle. Pour Charles Oursel, ce serait l'Évangéliste lui-même ${ }^{26}$. Pour Walter Cahn ${ }^{27}$, suivi par Yolanta Załuska ${ }^{28}$, Jean Wirth ${ }^{29}$ et Silke Tammen ${ }^{30}$, il s'agirait d'une véritable représentation d'Arius écrasé par Jean. D'autres spécialistes, comme Jennifer O'Reilly ${ }^{31}$ et Conrad Rudolph ${ }^{32}$, ont en revanche considéré l'image comme la représentation d'un moine idéalisé et l'enluminure comme une illustration de la lectio divina, selon l'invitation de saint Augustin à élever les sens à la compréhension totale et intime de l'Évangile de Jean.

Notre analyse, que nous avons présentée dans le cadre d'une étude sur l'iconographie de l'hérétique au Moyen Âge, tout en reprenant des idées déjà mises au jour par les études précédentes, propose un éclairage nouveau, qui voit dans la miniature une condamnation de la dialectique. Tout d'abord, nous avons penché pour identifier le moine avec Arius lui-même, la présence de l'hérésiarque au début de l'Évangile de Jean s'expliquant par le fait que le premier verset de cet Évangile était utilisé comme un argument de réfutation des doctrines ariennes. Cette identification n'explique toutefois pas tous les détails et la complexité de la formule iconographique suggère que d'autres significations se cachent derrière cette première observation. Notamment, à une observation attentive, on note que le personnage saisi par l'aigle est un moine seulement en apparence, car, sous sa tunique, des vêtements divers apparaissent. Il est possible ainsi d'y reconnaître la figure du faux moine, image souvent présente dans la 
littérature monastique. D’autres détails permettent de préciser son erreur. En premier lieu, remarquons que le personnage surmonté de l'aigle, tout en étant assis, est transporté en vol par celui-ci. En effet, d'une part, le moine est installé sur un coussin qui repose sur les deux bandes verticales posées sur le dos du lion situé dans la partie inférieure de l'image, alors que ses pieds reposent sur une feuille se soulevant comme un repose-pieds. D'autre part, les ailes ouvertes de l'aigle font indubitablement allusion au vol. Cette ambiguïté dans la position du moine, assis et transporté en vol en même temps, confirme sans doute l'interprétation de J. O'Reilly et de C. Rudolph selon lesquels le moine est pris dans la lecture de Jean qui lui ravit les sens, selon l'invitation de saint Augustin. Les paroles du Père de l'Église dans son commentaire de l'incipit de l'Évangile selon Jean s'adaptent en effet très bien à l'image. Après avoir insisté sur la nécessité pour les lecteurs de l'Évangile d'élever leurs sens, il ajoute : «Vous levez vos yeux et les sens de votre corps vers nous, et pourtant ce n'est pas même vers nous (...), c'est vers l'Évangile lui-même, vers l'Évangéliste lui-même ; mais votre cœur, c'est vers le Seigneur que vous le levez pour qu'il le remplisse ${ }^{33}$." L'enluminure dans son ensemble peut donc être lue sans doute comme une exhortation, faite aux moines, à la lecture contemplative des Écritures. De cette manière, il est possible de comprendre quelle est l'erreur du personnage, puni par l'aigle : il est quelqu'un qui ne se dédie pas à la lecture contemplative de l'Évangile, souhaitée par Augustin, mais plutôt à la lecture qui était considérée comme opposée à celle-ci, à savoir la lecture analytique, appliquant la dialectique aux Écritures. L'application de la dialectique à la théologie, opérée par les philosophes, était en effet vivement critiquée et rapprochée de l'hérésie dès le $\mathrm{XI}^{\mathrm{e}}$ siècle dans divers centres monastiques de l'Europe entière ${ }^{34}$. La miniature apparaît ainsi comme une mise en garde contre ce type de lecture. Afin de souligner la nuisance de la philosophie, le "philosophe » de l'image est assimilé à Arius, à savoir l'hérétique par excellence.

15 L'importance de l'enluminure est d'autant plus évidente que celle-ci est le premier témoignage de l'aversion de Cîteaux envers la dialectique appliquée aux Écritures. C'est quelques décennies plus tard que les cisterciens seront en première ligne dans l'attaque contre des maîtres de la scolastique. Guillaume, abbé de l'abbaye bénédictine de SaintThierry (au nord de Reims) entre 1121 et 1135, devenu cistercien en 1139, par peur des théories d'Abélard, les transmet à Bernard de Clairvaux et à Geoffroy, évêque de Chartres. Bernard accusa Abélard lors du concile de Sens, en 1140 ou 1141, et ce dernier fut condamné à la rétractation de ses propos et à l'enfermement dans un monastère ${ }^{35}$. L'enluminure démontre donc la façon dont la communauté de Cîteaux, en parfaite cohérence avec le désir de retour à la règle bénédictine, qui avait poussé ses fondateurs à quitter Molesmes, définit le bon comportement du moine en critiquant les mauvais moines : cette miniature montre de manière puissante l'attachement de la communauté à la tradition monastique et critique une pratique nouvelle, étrangère à la tradition, assimilée pour cela à une pratique "hérétique ». En même temps, elle témoigne d'une réflexion profonde sur l'hérésie arienne qui est évoquée et actualisée ${ }^{36}$.

16 L'image d'Arius se révèle donc une source importante pour la compréhension de certains idéaux de la communauté primitive des moines de Cîteaux. Nous nous proposons de poser la même problématique et d'appliquer la même méthode d'analyse dans l'étude des autres enluminures.

17 En conclusion, l'étude envisagée est susceptible de contribuer à l'exploration d'aspects importants de l'histoire primitive de Cîteaux, à partir de l'étude iconographique des 
enluminures de la Bible d'Étienne Harding. Elle visera donc à restituer l'histoire d'une communauté et d'une époque et à reconstruire ainsi la place de l'abbaye au sein des grandes mutations historiques qui caractérisent l'Europe entre les $\mathrm{XI}^{\mathrm{e}}$ et $\mathrm{XII}^{\mathrm{e}}$ siècles, sur le fond de la réforme de l'Église, de la suite de la Querelle pour les Investitures et de l'affirmation progressive des pouvoirs royaux capétien et anglais.

\section{NOTES}

1. .Pour l'étude codicologique de la Bible et sa datation, voir Y. ZAŁUSKA, L'enluminure et le scriptorium de Cîteaux au XIIe siècle, Cîteaux, 1989, p. 63-73. Une notice complète sur cette Bible se trouve aussi dans Y. ZAŁUSKA, Manuscrits enluminés de Dijon, Paris, 1991, p. 49-56.

2. D'autres manuscrits, pour lesquels la date n'a pas été établie avec précision, sont toutefois datables du premier quart du XIIe siècle : leur datation se fonde sur leurs ressemblances avec l'écriture et les enluminures de la Bible d'Étienne Harding et des Moralia in Job. Il s'agit des manuscrits 145, 146 (fol. 10-141) et 147, contenant les Enarrationes in Psalmos de saint Augustin, le ms 158, contenant le De civitate Dei, le 165 contenant des œuvres de Jean Cassien et de Grégoire le Grand, etc. Pour la présentation de ces manuscrits et d'autres codices enluminés à Cîteaux au cours du XIIe siècle, voir Y. ZAŁUSKA, Manuscrits..., ibid., p. 49-126.

3. Novum Monasterium est la dénomination la plus ancienne de l'abbaye de Cîteaux, qui subsiste jusqu'à 1119. Le nom Cistercium est toutefois utilisé avant cette date de manière sporadique. Pour l'usage des termes Novum Monasterium et Cistercium, se reporter à J. MARILIER, Chartes et documents concernant l'abbaye de Cîteaux : 1098-1182, Rome, 1961, p. 24-26.

4. Pour la manière dont la Bible était perçue et vécue dans les monastères, voir M.-C. CHARTIER, «Présence de la Bible dans les Règles et Coutumiers », in P. RICHÉ et G. LOBRICHON (dir.), Le Moyen Âge et la Bible, Paris, 1984.

5. Sur les circonstances dans lesquelles les Bibles viennent à faire partie des possessions des monastères de récente fondation, voir W. CAHN, La Bible romane. Chefs-d'œuvre de l'enluminure, Fribourg, 1982, p. 243-246. Dans de nombreux cas, les Bibles sont copiées dans les scriptoria des nouvelles abbayes mêmes. Pour une estimation de la présence de la Bible dans les bibliothèques médiévales, cf. P. PETITMENGIN, «La Bible à travers les inventaires de bibliothèques médiévales ", in P. RICHÉ et G. LOBRICHON (dir.), Le Moyen Âge..., ibid., p. 31-53. Il faut également remarquer que la copie des manuscrits au Moyen Âge n'est pas une corvée, mais un intense exercice spirituel qui pouvait servir à décharger les scribes de leurs péchés ; cf. W. CAHN, La Bible..., ibid., p. 245-246.

6. Il faut sans doute nuancer l'idée d'une pauvreté extrême de l'abbaye de Cîteaux dans les premières années de son existence. Certaines sources ont en effet tendance à l'accentuer afin de souligner l'importance de l'arrivée de Bernard de Clairvaux dans l'abbaye en 1113. Il est certain, en revanche, que l'abbatiat d'Albéric, entre 1099 et 1109, ne correspond pas à une période de richesse et d'expansion: après les premières donations de la part du duc de Bourgogne en 1098-1099, l'abbaye ne recevra, en effet, plus aucune donation temporelle jusqu'à 1109. Il est donc possible que la plupart des moines partaient du monastère, ce qui rendait le travail de mise en valeur des terres plus lourd aux autres et l'accession à des nouvelles terres inutile ; pour ces données, cf. J.-B. AUBERGER, L'unanimité cistercienne primitive : mythe ou réalité ?, Achel, 1986 (Studia et documenta, 3), p. 138-142. Pour une discussion sur les sources et sur les enjeux liés à la réécriture des origines de Cîteaux, voir C. CABY, «De l'abbaye à l'ordre. Écriture des origines et 
institutionnalisation des expériences monastiques XIe-XIIe siècle ", Mélanges de l'École française de Rome, 115 (2003), p. 235-267.

7. Pour le texte latin du Monitum et sa traduction française, ainsi que pour les principes de critique textuelle mis en œuvre dans la Bible, se reporter à M. CAUWE, «La Bible d'Étienne Harding. Principes de critique textuelle mis en œuvre aux livres de Samuel », Revue bénédictine, 103 (1993), p. 414-444, part. p. 416-417. Pour quelques remarques supplémentaires à ce sujet, voir également G. DAHAN, L'exégèse chrétienne de la Bible en Occident médiéval. XIIe-XIVe siècle, Paris, 1999, p. 168-171. Remarquons que, selon certains auteurs, Étienne Harding ne se serait pas limité à la révision du texte biblique, mais aurait également réalisé les enluminures ; cf. par exemple, J. PORCHER, «L'enluminure cistercienne », in A. DIMIER et J. PORCHER, L'art cistercien, Saint-LégerVauban, 1962, p. 320-325. Bien que certaines enluminures montrent un style très proche du style anglais de l'époque - cf. par exemple, la figure du moine au fol. $83 \mathrm{v}$ du ms 15 -, cette hypothèse ne peut pas être vérifiée.

8. Ce phénomène de "retour aux sources" est bien décrit dans A. VAUCHEZ, La spiritualité du Moyen Âge occidental. VIIIe-XIIIe siècle, Paris, 1994, p. 73-79 (pour le phénomène général) et p. 91-95 (en particulier sur les cisterciens). La Réforme ecclésiastique des XIe et XIIe siècles, surtout dans ses implications artistiques, est au centre, depuis quelques années, de la réflexion des spécialistes. On peut voir, à ce propos, J. ENCKELL JULLIARD et S. ROMANO (dir.), Roma e la Riforma gregoriana. Tradizioni e innovazioni artistiche (XI-XII secolo), Rome, 2007. Nous avons déjà eu l'occasion de faire une introduction générale aux relations entre la Réforme et l'art, dans A. TRIVEllone, «L'arte e la Riforma ecclesiastica dell'XI secolo », in U. ECo (dir.), Il Medioevo, Milan, 2009, p. 493-516.

9. L'histoire des débuts de Cîteaux est largement débattue, notamment dans la critique française et américaine. Parmi les ouvrages principaux sur les origines de Cîteaux et de l'ordre cistercien, signalons: J.-B. AUBERGER, L'unanimité cistercienne..., op. cit.; C.B.BOUCHARD, Sword, miter, and cloister: nobility and the church in Burgundy, 980-1198, Ithaca/Londres, 1987 ; M. G. NEwMAN, The Boundaries of Charity: Cistercian Culture and Ecclesiastical Reform 1098-1180, Stanford, 1996; C. HOFFMAN BERMAN, The Cistercian evolution: The Invention of a Religious Order in Twelth-Century Europe, Philadelphia, 1999 [mais l'hypothèse qu'elle avance sur la naissance de l'ordre cistercien au XIIe siècle avancé est débattue: voir à ce propos le compte rendu de C. CABY, dans Revue d'histoire ecclésiastique, 99 (2004), p. 830-832]; Réformes et continuité dans l'ordre de Cîteaux: de l'étroite observance à la stricte observance: actes du Colloque Journées d'histoire monastique, Saint-Mihiel 2-3 octobre 1992, Brecht, 1995 ; Unanimité et diversité cisterciennes : filiations, réseaux, relectures du XIIe au XVIIe siècle, Saint-Étienne, 2000. Pour l'édition des sources cisterciennes des débuts, voir C. WADDELL, Narrative and legislative texts from early Cîteaux. Latin text in dual edition with english translation and notes, Cîteaux, 1999 (Commentarii cistercienses), alors que les chartes et les documents relatifs à Cîteaux jusqu'à 1182 sont édités dans J. MARILIER, Chartes et documents..., op. cit. Une riche bibliographie sur l'histoire de Cîteaux est indiquée dans C. CABY, "De l'abbaye à l'ordre... », op. cit.

10. La bibliographie sur l'enluminure cistercienne est très vaste. Sans prétention d'exhaustivité, citons, parmi les études principales, C. OURSEL, «Les manuscrits à miniatures de la Bibliothèque de Dijon », Bulletin de la Société française de reproductions des manuscrits à peintures, Paris, 1923 ; ID., La miniature du XIIe siècle à l'abbaye de Cîteaux d'après les manuscrits de la bibliothèque de Dijon, Dijon, 1926 ; ID., "La Bible de saint Étienne Harding et le scriptorium de Cîteaux ", Cîteaux in de Nederlanden, 6 (1955), p. 162-171; ID., «Les principes et l'esprit des miniatures primitives de Cîteaux », Cîteaux in de Nederlanden, 10 (1959), p. 34-43 ; ID., Miniatures cisterciennes (1109-1134), Mâcon, 1960 ; ID., «La genèse des manuscrits primitifs de l'abbaye de Cîteaux sous l'abbatiat de saint Étienne Harding ", Mémoires de l'Académie des sciences, arts et belles-lettres de Dijon, 114 (1961), p. 43-52 ; J. PORCHER, «L'enluminure... », op. cit.; A. M. ROMANINI, « Il "Maestro dei Moralia" e le 
origini di Cîteaux", Storia dell'arte, 32/34 (1978), p. 221-245; Y. ZALUSKA, L'enluminure et le scriptorium..., op. cit., p. 75-85; M. PARSONS LILLICH (éd.), Studies in cistercian art and architecture, Kalamazoo, 1987 (Cistercian studies series, 89) ; A. VANNUGLI, « Il "secondo maestro" di Cîteaux e la sua attività in Borgogna », Arte medievale, 2/3 (1989), 2, p. 51-72; Y. ZAŁUSKA, "L'enluminure cistercienne au XIIe siècle », in Bernard de Clairvaux. Histoire, mentalités, spiritualité. Colloque de LyonCîteaux-Dijon,1992 (Sources chrétiennes, 380), p. 271-285 ; J.-B. AUBERGER, L'unanimité cistercienne..., ibid., p. 190-195 ; N. STRATFORD, « A Romanesque marble altar-frontal in Beaune and some Cîteaux manuscripts", in Studies in Burgundian romanesque sculpture, Londres, 1998 ; M. PORTELLI, «Les Morales sur Job de Saint-Grégoire Le Grand provenant de l'abbaye de la Ferté-sur-Grosne et la production livresque de l'abbaye de Cîteaux au XIIe siècle ", Annales de Bourgogne, 75/1 (2003), p. 81-92.

11. Quelques enluminures ont toutefois fait l'objet d'études ponctuelles. Outre l'image de l'incipit de l'Évangile selon Jean (ms 15, fol.56v), que nous allons examiner plus loin, on peut rappeler l'image du Christ en Majesté entre l'Église et la Synagogue (ms 14, fol. 50), déjà étudiée dans M.-L. THÉREL, "L'origine du thème de la Synagogue répudiée ", Scriptorium, 25 (1971), p. 285-290 et D. J. ReILLY, « Picturing the Monastic Drama : Romanesque Bible illustrations of the Song of Songs ", Word \& image, 17 (2001), p. 389-400. De plus, les scènes concernant la vie de David (ms 14, fol. 13) ont fait l'objet de l'analyse de Y. Załuska (L'enluminure et le scriptorium..., op. cit., p. 85-105).

12. Nous refuserons, ainsi, la démarche de certains spécialistes postulant que l'enlumineur copie forcément des modèles tirés des représentations ou cycles précédents: pour expliquer l'originalité des formules iconographiques, ils supposent souvent que ces modèles sont perdus. Cette perspective réduit inévitablement l'importance des innovations iconographiques employées dans la Bible et empêche d'en comprendre le vrai sens. L'approche exclusivement vouée à rechercher des modèles pour les miniatures, a été critiquée, très à propos, par Y. Załuska (L'enluminure et le scriptorium..., ibid., p. 104-105).

13. Pour un panorama de l'illustration biblique aux XIe et XIIe siècles, voir W. CAHN, La Bible..., op. cit.

14. Cf. supra note 11 .

15. C'est l'approche de P. Buc, L'ambiguité du livre. Prince, pouvoir, et peuple dans les commentaires de la Bible au Moyen Âge, Paris, 1994. L'influence de la Bible sur la vie politique du haut Moyen Âge a été en revanche étudiée dans P. RICHÉ, «La Bible et la vie politique dans le haut Moyen Âge », in P. RICHÉ et G. LOBRICHON (dir.), Le Moyen Âge..., op. cit., p. 385-400.

16. D. J. REILLY, The Art of Reform in Eleventh-Century Flanders: Gerard of Cambrai, Richard of SaintVanne and the Saint-Vaast Bible, Leiden, 2006.

17. U. NILGEN, « Historischer Schriftsinn und ironische Weltbetrachtung : Buchmalerei im frühen Cîteaux und der Stein des Anstoßes ", in K. ELM (éd.), Bernhard von Clairvaux : Rezeption und Wirkung im Mittelalter und in der Neuzeit, Wiesbaden, 1994 (Wolfenbütteler Mittelalter-Studien, 6), p. 67-140 ; EAD., "Sacra Scrittura e mondo feudale : il contributo delle immagini ", in Chiesa e mondo feudale nei secoli X-XII. Atti della dodicesima Settimana internazionale di studio (Mendola, 24-28 agosto 1992), Milan, 1995, p. 553-566. On regrettera, en particulier, l'insistance d'Ursula Nilgen sur une prétendue « ironie » des scènes, évoquée de manière anachronique et gratuite : l'auteur ne fait en effet aucun effort pour essayer de vérifier que cette idée d'« ironie » s'intègre dans les catégories de la mentalité médiévale et ne quitte pas sa place d'observatrice moderne.

18. À ce propos, il faut remarquer que, bien que la datation des sources primitives cisterciennes (Exordium Parvum, Exordium Cistercii, Charta Charitatis dans ses trois versions) soit encore de nos jours débattue, aucune des hypothèses émises n'a jamais envisagé, pour aucune des sources en question, une date antérieure à 1111, date où la Bible fut avec toute vraisemblance achevée ; voir, pour les sources relatives à Cîteaux, C. WADDELL, Narrative and legislative texts..., op. cit. 
19. Y. ZAŁUSKA, L'enluminure et le scriptorium..., op. cit., p. 68 et fig. 7a (p. 197). Selon l'auteur, le livre de Job aurait, dans un premier temps, été oublié. D'autres corrections importantes, qui ont impliqué l'ajout ou le remplacement de feuillets, semblent faites après la réalisation du manuscrit pour corriger des fautes de copie ; cf. ZAŁUSKA, L'enluminure et le scriptorium..., ibid., p. 68-69.

20. C'est pourquoi l'emplacement de cette image serait le fruit, selon Y. Załuska, de l'inspiration à des modèles peints représentant la vie de David ; cf. ZAŁUSKA, L'enluminure et le scriptorium..., ibid., p. 87. Cette interprétation nous semble toutefois réductrice. Sur l'iconographie des cycles de David, voir G. SUCKALE REDLEFSEN, Die Bilderzyclen zum Davidleben von den Anfängen bis zum Ende des 11. Jahrhunderts, Munich, 1972.

21. Cf. ZALUSKA, L'enluminure et le scriptorium..., ibid., p. 87.

22. Ainsi, par exemple, Saverio Lomartire, en étudiant le rinceau peuplé de la porte du zodiaque à Sagra di San Michele, insiste sur son caractère cathartique: il serait une invitation faite à l'homme de maîtriser ses passions humaines avant d'entrer dans le lieu sacré ; cf. S. LOMARTIRE, «Testo e immagine nella Porta dello Zodiaco", in Dal Piemonte all'Europa: esperienze monastiche nella società medievale, Turin, 1988, p. 431-474. Selon Jean-Pierre Caillet, le rinceau peuplé sur quelques candélabres médiévaux apparaît avoir été mis à profit pour exprimer le destin de l'homme dans sa chute et sa rédemption : les rinceaux peuplés abritant la lutte entre animaux et homme illustreraient la première phase (celle de la chute) et feraient référence, grâce aussi à une insistance sur le rôle négatif de la forêt, aux défis que l'humanité rencontre lors de sa vie terrestre ; cf. J.-P. CAILLLET, « "Et magnae silvae creverunt...” Observations sur le thème du rinceau peuplé dans l'orfèvrerie et l'ivoirerie liturgiques aux époques ottonienne et romane ", Cahiers de civilisation médiévale, 38 (1995), p. 23-33. Harald Wolter-von dem Knesebeck, étudiant les enluminures à l'incipit du premier psaume dans le psautier d'Élisabeth de Cividale del Friuli, identifie dans les rinceaux peuplés les activités humaines (comme la chasse) éloignées de la contemplation divine; cf. H.W. VON DEM KNESEBECK, Der Elisabethpsalter in Cividale del Friuli: Buchmalerei für den Thüringer Landgrafenhof zu Beginn des 13. Jahrhunderts, Berlin, 2001. Nous avons eu l'occasion de nous pencher sur la signification des rinceaux peuplés sur les lieux de passage, dans une communication au titre «Hommes, feuillages et bêtes des passages: valeur apotropaïque des sculptures à côté des seuils au Moyen Âge ", présentée lors de la table ronde "Images et passages à l'époque médiévale », organisée par Dominique Donadieu-Rigaut (Auxerre, Centre d'études médiévales, 19-20 juin 2008).

23. Pour l'iconographie de David, se reporter à R. L. WYSS, "David », in Lexikon der christlichen Ikonographie, Rome/Fribourg/Bâle/Vienne, 1968-1976, t. 1 (1968), col. 477 - 490.

24. Observons, toutefois, que le premier feuillet du volume a été amputé. Puisque le fol.2r commence par le deuxième verset du livre de Job (et erat ille vir simplex et rectus), le premier feuillet contenait vraisemblablement le prologue et l'incipit du livre de Job. On ne peut pas exclure qu'il présentait également une miniature, qui serait, dans ce cas, la première du deuxième volume ancien. Remarquons, de plus, que six initiales avec des végétaux imbriquant des animaux se retrouvent également plus loin dans les manuscrits 14 et 15, qui formaient l'ancien deuxième volume de la Bible. Observons, cependant, que dans deux cas, la présence de ces enluminures s'explique par d'autres considérations. Ainsi, dans le manuscrit 14, une initiale avec un centaure imbriqué dans des rinceaux végétaux se trouve au fol. 14. L'initiale est pourtant le «B» de l'incipit du premier psaume («Beatus vir»), traditionnellement décoré par des rinceaux, peuplés ou non. La pertinence de la présence du rinceau peuplé dans les initiales du premier psaume et sa signification a été expliquée par Von Knesebeck (Der Elisabethpsalter..., op. cit.). Une enluminure à rinceaux végétaux du manuscrit 15 consiste dans une initiale $\mathrm{E}$ (fol. $3 \mathrm{v}$ ), montrant un griffon et un lion dans des rinceaux végétaux. La disposition de ces animaux - le griffon dans la partie supérieure de la lettre et le lion en bas - nous fait toutefois pencher pour 
une diverse signification de cette représentation, qui, plus qu'à la forêt et à la nature, semblerait faire allusion à une représentation cosmique, le lion étant, pour les commentateurs médiévaux, le roi de la terre et le griffon unissant en lui les qualités de roi de la terre et du ciel. Les autres initiales à rinceaux apparaissent au manuscrit 14, fol.136v (incipit de l'Ecclésiastique) et au manuscrit 15, fol. 94, 105 et 108v (épîtres paulines). Certaines de ces dernières enluminures (cf., par exemple, ms 15, fol. 94) se rapprochent, pour leur austérité figurative, de quelques initiales des Enarrationes in Psalmos d'Augustin (Dijon, BM, ms 145 et 147). Pour ces dernières, Y. Załuska propose qu'elles puissent avoir été réalisées après 1113-1115, suite à des discussions éventuelles sur la simplicité dans l'art engendrées par le passage de Bernard à Cîteaux; cf. Y. ZAŁUSKA, L'enluminure et le scriptorium..., op. cit., p. 81. Il sera donc à vérifier, avec un examen attentif, si les initiales des lettres paulines font effectivement partie de la décoration originaire de la Bible.

25. Cf. supra, note 11.

26. C. OURSEL, La miniature au XIIe siècle..., op. cit., p. 27 ; J. WIRTH, L'image à l'époque..., op. cit., p. 214.

27. W. CAHN, "A defense of the Trinity in the Cîteaux Bible», Marsyas, 11 (1962-1964), p. 58-62 (rééd. dans ID., Studies in Medieval Art and Interpretation, Londres, 2000, p. 1-14); ID., « Heresy and the Interpretation of Romanesque Art », in N. STRATFORD(éd.), Romanesque and Gothic. Essays for George Zarnecki, Woodbridge, 1987, p. 27-33, part. p. 32 ; ID., La Bible..., op. cit., p. 142-143.

28. Y. ZAŁUSKA, L'enluminure et le scriptorium..., op. cit., p. 110 -111 ; EAD., Manuscrits, enluminés..., op. cit., p. 54 .

29. J. WiRTh, L'image à l'époque..., op. cit., p. 214 ;

30. S. TAMMEN, « Disput und Triumph. Zum Bild des Häretikers in der mittelalterlichen Kunst ", Frühmittelalterliche Studien, 35 (2001), p. 407-430, ici p. 415-416.

31. J. o'REILLY, «St John as a Figure of the Contemplative Life: Text and Image in the Art of the Anglo-Saxon Benedictine Reform ", in N. RAMSAY, M. SPARKS et T. TATTON-BRown (éd.), St Dunstan. His life, Times and Cult, Suffolk, 1992, p. 165-185, ici p. 177-178.

32. C. RudolPh, Violence and Daily Life. Reading, Art and Polemics in the Citeaux Moralia in Job, Princeton, 1997, p. 88 et note 19 (p. 123).

33. AUGuStin, Homélies sur l'Évangile, p. 143.

34. Sur cette question, se reporter à l'étude d'A. CANTIN, Foi et dialectique au XIe siècle, Paris, 1997, ainsi qu'à l'ouvrage fondamental de H. FichtenAu, Heretics and Scholars in the High Middle Ages, 1000-1200, Pennsylvania State University, 1998 (1ère éd. allemande : Ketzer und Professoren : Häresie und Vernunftglaube im Hochmittelalter, Munich, 1992), surtout au chapitre 12, «Early scholasticism and Heresy ", p. 281-311.

35. Pour une analyse plus approfondie de l'image et la présentation d'une bibliographie détaillée sur les différentes questions abordées, nous renvoyons à A. TRIVELLONE, L'hérétique imaginé. Hétérodoxie et iconographie dans l'Occident médiéval, de l'époque carolingienne à l'Inquisition, Turnhout (à paraître en 2009), chap. 4.3 .

36. Sur la progressive actualisation du concept d'arianisme en Occident, je renvoie à A. TRIVELLONE, L'hérétique imaginé..., ibid., chap. 4.5 .

\section{INDEX}

Index géographique : France/Cîteaux

Mots-clés : Etienne Harding, Bible 